\title{
A hybrid approach for generating ultra-short bunches for advanced accelerator applications
}

\author{
D. Stratakis
}

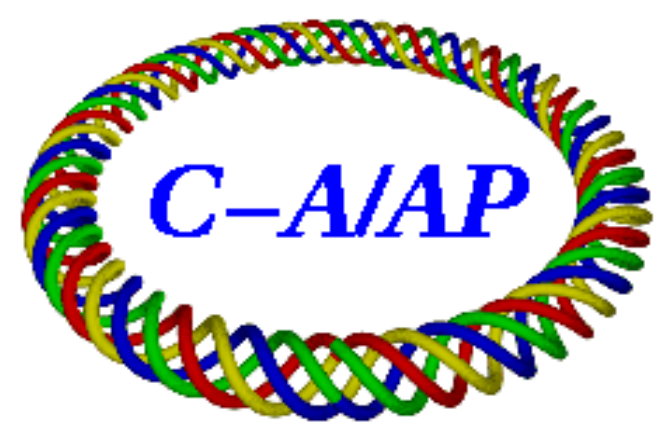

Collider-Accelerator Department Brookhaven National Laboratory

Upton, NY 11973

\section{U.S. Department of Energy}

\section{Office of Science, Office of Nuclear Physics}

Notice: This document has been authorized by employees of Brookhaven Science Associates, LLC under Contract No. DE-SC0012704 with the U.S. Department of Energy. The United States Government retains a nonexclusive, paid-up, irrevocable, world-wide license to publish or reproduce the published form of this document, or allow others to do so, for United States Government purposes. 


\title{
A hybrid approach for generating ultra-short bunches for advanced
}

\section{accelerator applications}

\author{
Diktys Stratakis \\ Brookhaven National Laboratory, Upton NY 11973, USA
}

(Dated: September 01, 2015)

\begin{abstract}
Generation of electron beams with high phase-space density, short bunch length and high peak current is an essential requirement for future linear colliders and bright electron beam sources. Unfortunately, such bunches cannot be produced directly from the source since forces from the mutual repulsion of electrons would destroy the brilliance of the beam within a short distance. Here, we detail a beam dynamics study of an innovative two-stage compression scheme that can generate ultra-short bunches without degrading the beam quality. In the first stage, the beam is compressed with an advanced velocity bunching technique in which the longitudinal phase space is rotated so that electrons on the bunch tail become faster than electrons in the bunch head. In the second stage, the beam is further compressed with a conventional magnetic chicane. With the aid of numerical simulations we show that our two-staged scheme is capable to increase the current of a $50 \mathrm{pC}$ bunch to a notable factor of 100 while the emittance growth can be suppressed to $1 \%$ with appropriate tailoring of the initial beam distribution.
\end{abstract}




\section{INTRODUCTION}

Electron beams with low transverse normalized emittance (sub- $\mu \mathrm{m}$ scale), short bunch length (at the sub-ps scale), and high peak current (up to thousands of amperes) is a key requirement in high-brightness light sources [1], plasma wave accelerations [2] and linear colliders [3]. Unfortunately, such bunches cannot be produced from the source directly, since forces from the mutual repulsion of electrons would destroy the brilliance within a short distance [4]. Instead, they are obtained by first accelerating a low current beam to relativistic energies and subsequently compressing the bunch length by several orders of magnitude to increase the peak current.

In most designs, the compression is achieved in magnet chicanes, where particles with different energies have different path lengths, so that a bunch with an energy distribution correlated with longitudinal particle position can shrink in length [5]. A successful compression system is one that compresses the bunch longitudinally while preserving the beam's transverse phase-space area, referred to as transverse beam emittance. In most cases, however, as a bunch propagates through the bends of a compressor, the tail-head interaction due to coherent synchrotron radiation (CSR) can induce increased beam energy spread and transverse phasespace dilution that can severely degrade the downstream performance. In addition, CSR interaction is known to generate a micro-bunching instability in which large-amplitude current modulations can grow from small-amplitude, short-wavelength variations in the initial current profile [6-8].

Several techniques have been developed to mitigate the CSR effect [9-11]. For instance, utilizing asymmetry in a chicane design has shown success in partial nullification of emittance dilution when compared to the standard symmetric designs. Additionally, it has been shown that 
in multi-stage compression systems, allocating more to the initial bunch compressors while maintaining final compression, dampens the CSR effect in the final compressor where CSR is most detrimental. Though the techniques cited demonstrate effective reduction of the CSR they tend to require many additional magnetic elements which beside the cost constraints can also lead to further degradation of the longitudinal phase space and fall short of complete nullification.

A promising solution to avoid this problem, termed velocity bunching, has been proposed [12]. Velocity bunching (VB) relies on the phase slippage between the electrons and the rf wave that occurs during the acceleration of non-relativistic electrons. The key point is that compression and acceleration takes place at the same time within the same accelerator section, the initial following the gun, thus making this technique very attractive. Such type of bunching has been experimentally observed in laser driven rf electron sources [13, 14]. Over the last decade, numerous experimental works demonstrated further the unique compression ability of the VB method [15-18].

It is important to emphasize that utilizing VB for very high compression ratios can lead to substantial emittance growth and non-linear phase-spaces [19]. To circumvent this difficulty, we consider and present in this paper detailed numerical simulations of a innovative "two-stage" compression scheme. In the first stage, a mild compression is achieved with the VB technique. During the process, both beam and lattice parameters are properly tuned so that to maintain a near linear longitudinal phase space and avoid emittance growth. In the second stage, the beam is further compressed at fs-scale with a conventional magnetic chicane. Due to the initial VB, the chicane compresses the beam with minimum distortion from the CSR since the dipoles needed are of lower bending angle. With the aid of numerical simulations, we show that our two-stage 
scheme is capable to increase the current of a $50 \mathrm{pC}$ bunch to a notable factor of 100 while the emittance growth is suppressed to just $1 \%$ with appropriate tuning of the beam optics.

The outline of the paper is as follows: In Sec. II, we give an overview of the accelerator beamline components, in Sec. III we describe the velocity bunching process, and in Sec, IV we detail a beam dynamics study and present numerical results from modeling two-stage compression on the aforementioned channel. Finally, we present our conclusions in Sec. V.

\section{ACCELERATOR BEAMLINE OVERVIEW}

For our beam dynamics studies we consider the photo-injector beamline of the Brookhaven National Laboratory (BNL) Accelerator Test Facility (ATF) upgrade [20] which is shown schematically in Fig. 1. In brief, electron charges with variable charge $(\mathrm{Q} \leq 3.2 \mathrm{nC})$ are generated via photoemission from a metal photocathode located at the back plate of a 1.6 cell radiofrequency $(\mathrm{RF})$ operating at $2856 \mathrm{MHz}$. The gun is surrounded by a solenoid magnet S1 which is used to correct for the space-charge induced correlated emittance growth. If a magnetized beam is desired, a second solenoid S2 is turned on to provide a magnetic field on the cathode along with proper focusing. The electron bunches exiting the rf gun are then accelerated by two three meter long $2.856 \mathrm{GHz}$ (S-Band) traveling wave structures (linacs L1 and L2), to energies that can exceed $100 \mathrm{MeV}$ and potentially raise to $150 \mathrm{MeV}$. Downstream of the second acceleration section, is a set of three quadrupoles which can turn into skew magnets depending on the desired downstream distribution. The beamline also incorporates a four-bend magnetic bunch compressor (BC) which, consists of four rectangular dipoles with respective bending angles and the bending is assumed to be on the horizontal plane. During compressor operations, an energy-time correlation is imparted onto the electron bunch by operating the two travelling 
wave structures off-crest. The induced correlation allows for a change in the time of flight of the particles of the beam, effectively shortening it's bunch length, thereby increasing the current.

In the VB process, the longitudinal phase space rotation is based on a correlated timevelocity chirp in the electron bunch, so that electrons on the tail are faster than electrons in the bunch head. This rotation occurs inside a traveling rf wave of a long multi-cell $\mathrm{rf}$ structure which applies an off crest energy chirp to the injected beam as well as accelerates it. This is possible if the injected beam is slightly slower than the phase velocity of the rf wave so that when injected at the zero crossing field phase it slips back to phases where the field is accelerating, but is simultaneously chirped and compressed. To implement VB scheme for the ATF, the first linac section L1 was used as a buncher: its phase was varied and, for each phase setting, linac L2 was properly phased to maximize the beam energy with the BC turned off.

\section{SIMULATION RESULTS: VELOCITY BUNCHING}

The numerical simulations presented in this paper were carried out with ASTRA [21] and ELEGANT [22] codes. ASTRA is a macroparticle code based on a rotationally symmetric spacecharge algorithm and incorporates a detailed model for the traveling wave structure. The laser was modeled by a radially uniform transverse distribution with $0.25 \mathrm{~mm}$ radius. In the beamline of Fig. 1, ASTRA was used to simulate the beam dynamics from its formation at the photocathode up to the entrance of the quadrupole triplet with 200k macroparticles. Then, these macroparticles were used as input in ELEGANT, a well-established code for CSR simulation, and tracked through the remaining lattice.

In the simulation the drive laser was operated with a $~ 7.0$ ps FWHM long pulse, having a rms spot radius of $0.25 \mathrm{~mm}$. The bunch charge was $50 \mathrm{pC}$ giving a maximum slice current of about $15 \mathrm{~A}$ without compression. At the gun exit the beam energy was $5.0 \mathrm{MeV}$ corresponding to 
a peak field on the cathode of $110 \mathrm{MV} / \mathrm{m}$. When the beam was accelerated on crest, with an accelerating field amplitude of $20 \mathrm{MV} / \mathrm{m}$ in the first and $30 \mathrm{MV} / \mathrm{m}$ in the second section, the final energy was $116.5 \mathrm{MeV}$ with an energy spread of $0.02 \%$. The rms bunch length at the linac exit was $1.13 \mathrm{ps}$, with a normalized transverse emittance of $0.65 \mu \mathrm{m}$ in both planes with the gun solenoid set to $0.19 \mathrm{~T}$ (the magnetized solenoid $\mathrm{S} 2$ was off). The beam parameters are summarized in more detail in Table I.

Figure 2(a) shows the simulated rms bunch length $\sigma_{l, f}$ after compression versus the injection phase $\varphi$ of the first linac L1. Figure 2(b) shows the corresponding rms compression factor C, which is defined by the bunch length after on crest acceleration divided by the bunch length after compression. As shown in Fig. 2 to induce significant compression, the beam injection in the compressor has to be delayed by more than $80^{\circ}$. Peak bunch compression occurs at a phase shift of $-89^{0}$ forward of crest. Figure 3 displays the energy as function of the compression phase. Clearly, an unfortunate drawback of VB is that the final beam energy reduces by $20 \%$ or more compared to on-crest acceleration. Quantitatively, at the peak injection phase the beam energy reduces to $81.3 \mathrm{MeV}$ and the energy spread grows to $0.4 \%$. The corresponding shortest rms bunch length is $42.3 \mathrm{fs}$, limited by the longitudinal beam emittance.

Figure 4 shows the simulated evolution of the emittances along the channel for three different compression factors: $\mathrm{C}=17.4,9.1$, and 3.5. Figure 4 clearly shows that the induced emittance growth is related to the degree of compression. In particular, when bunch compression is significant $(\mathrm{C}>9)$ the emittance grows by a factor of two or more while at moderate compressions the emittance remains relatively flat. Emittance growth in velocity bunching has been shown to be compensated by placing a long solenoid around the travelling wave structure. 
However, this solution is followed by serious alignment challenges and thus the purpose of this work is to identify an alternative mitigation strategy.

Figure 5 displays several current profiles for different compression strengths. Note that depending on the compression phase, the peaked currents are ranging from a few tenths of Amps up to $1.6 \mathrm{kA}$. In direct agreement with previously conducted experiments in Ref. 19, the current distributions display asymmetric profiles with long tails that can be particularly problematic if further compression is foreseen downstream. They are usually associated with longitudinal phase space non-linearities caused by the rf fields or by energy-velocity correlations or a combination of those two $[16,19]$. To illustrate evidence of such non-linearities we plot in Fig. 6(a) the longitudinal phase-phase for the maximum compression case $(C=27.9)$. It is interesting to note that as $\mathrm{C} \leq 5$ such deleterious effects tend to vanish [see Fig. 6(b)] suggesting that the VB method is more suitable for moderate compressions.

\section{SIMULATION RESULTS: A HYBRID SOLUTION}

As noted earlier, strong compression with VB introduces transverse emittance growth and nonlinearities on the longitudinal phase-space that must be dealt with through the rest of the beamline. To circumvent this issue, we propose a two-staged compression scheme that employs both velocity bunching and magnetic bunch compression. With this hybrid scheme, linac L1 can operate off its optimal phase and further from the zero-crossing. Although VB still introduces energy spread and other nonlinearities to the bunch, since compression is not maximal the effects are much less destructive and the beam maintains a linear enegy-time correlation [see Fig. 6(b)].

Subsequently, the rotation through BC fully compresses the bunch but with less distortion since the dipoles of the compressor can now be of a lower bending angle. 
In our simulation we have added freedom of having tunable $\mathrm{R}_{56}$ for the magnetic compressor allowing us to have an additional optimization parameter for the second part of compression. With this, we can match an optimal $\mathrm{R}_{56}$ to each off crest phase of $\mathrm{L} 1$ and scan for the combination that gives us optimal bunch compression - figures of merit for the optimal L1 phase- $\mathrm{R}_{56}$ combination are the peak current, rms bunch length and final emittance. Based on our numerical findings, for a $50 \mathrm{pC}$ bunch that is compressed by a factor of 4.1 (optimal phase of $\left.\mathrm{L} 1=-84^{0}\right)$, the optical chicane parameters are listed in Table II. Note that the bending angle of the compressor is just $7.4^{0}$ and $\mathrm{R}_{56}$ is $-1.1 \mathrm{~cm}$.

The evolution of the transverse emittance along the channel for a $50 \mathrm{pC}$ beam is shown in Figure 7(a). Note that for this simulation the current of the quadrupole triplet was zero resulting on a round beam at the chicane entrance. Figure 7(b) displays the current profile at OP while Fig. 7(c) shows the corresponding distribution in configuration space. By carefully examining Fig. 5(d) and Fig. 7(b) the following points are noteworthy: First, the final bunch length with the twostage-scheme is at least 2 times smaller than with VB only. Moreover, the current profile lacks the long tail, which is a direct consequence of the suppression of nonlinearities in the longitudinal phase space. As anticipated, the weak chicane reduced the induced horizontal emittance growth from CSR to $\sim 25 \%$ compared to a threefold increase with a single compression scheme. According to our simulations, the growth occurs mostly within the third and fourth magnets where the beam is already compressed and the peak current is high. The growth in transverse emittance reflects the fact that CSR induces a variation of the particle energy that is correlated with longitudinal coordinate along the bunch. As a consequence, different bunch slices are deflected with different angles in a bending magnet, and this deflecting error distorts the transverse phase space [see Figure 7(d)]. Based on the results in Fig. 7, our 
hybrid technique increased the bunch current by a notable factor of 100 (from $15 \mathrm{~A}$ to $1500 \mathrm{kA}$ ) while it reduced rms bunch length to just 15 fs.

Next we examine the case of flat beam as it transverses the same magnetic bunch compressor as the previous beam. The motivation of examining flat beams, i.e. beams with large transverse emittance ratios, is that such beams have proposed in the context of linear colliders and some novel electron-beam-based light sources. For instance, in the case of a linear e+e- collider a flat beam at the interaction point reduces the luminosity disruption caused by bremsstrahlung [23, 24]. As we will show shortly the flat beam has another property: under careful compression it can preserve its emittance.

In order to generate a flat beam we immerse the photocathode in an axial magnetic field and thereby introduce an average canonical angular momentum $\langle L\rangle=e B_{0} \sigma_{c}^{2}$ where is $e$ the electron charge, $B_{0}$ is the axial magnetic field on the photocathode surface, and $\sigma_{c}$ is the rootmean-square (rms) transverse size of the drive-laser spot on the photocathode. As the beam exits the solenoidal field the angular momentum is purely kinetic resulting in a beam coupled in the two transverse planes. A set of three skew quadrupoles can apply the torque necessary to remove the angular momentum of the beam and to break the symmetry between the $\mathrm{x}$ and $\mathrm{y}$ plane and thus generate an asymmetric beam between the two transverse planes; see a more detailed analysis in Refs. 24, 25. Following the procedure described in Ref. 25, the strengths of the quadrupoles are chosen such as to minimize the figure of merit $\mathrm{FOM}^{2}=\Sigma_{13}^{2}+\Sigma_{14}^{2}+\Sigma_{23}^{2}+\Sigma_{24}^{2}$ at the triplet exit where $\Sigma_{i j}$ is the element of the covariance matrix $\Sigma$. The resulting quadrupole magnet settings were then used used in ELEGANT to track the beam throughout the skew triplet.

Figure 8(a) displays the emittance evolution of a flat beam. The assumed field on the cathode is $1000 \mathrm{G}$ while the focusing strengths of the three skew quadrupoles are $\kappa_{1}=10, \kappa_{2}=20$, and 
$\kappa_{3}=100 \mathrm{~m}^{-2}$. Note that a skew quadrupole is considered here a normal quadrupole (as shown in Fig. 1) but rotated $45^{\circ}$ in the transverse $(x-y)$ plane. As before, the phase of the first linac L1is set at $-84^{0}$ so that to provide an initial bunch compression of 4.1 via velocity bunching. In addition, the chicane parameters are those listed in Table II. As one can see from Fig. 8, the above set of parameters generates a flat beam with a transverse emittance ratio $\varepsilon_{x} / \varepsilon_{y}$ of 30 . A salient feature of such asymmetric beam is that the induced horizontal emittance growth from CSR is less than $1 \%$. The vertical emittance remains also stable, at just $0.17 \mu \mathrm{m}$, suggesting that a flat beam can offer a promising solution to maintain low emittance at one dimension. Figure 8(b) shows the beam profile at the chicane exit which as before has an rms bunch length of 15 fs. The corresponding distributions in configuration and phase space are shown in Figs. 8(c) and 8(d), respectively. Conversely to the results of Fig. 7, the smearing effect is completely absent and the phase-space retains its elliptical shape. The reason that the magnetized beam could mitigate the emittance growth and avoid phase-space distortion is as follows. Based on our simulations, the asymmetric beam maintains a larger bunch length at the entrance of the fourth dipole (where the CSR is expected to be the greatest) compared to the round beam and the minimum bunch length is only achieved at the end of the fourth dipole magnet, so the angular spread of the beam introduced by the CSR induced energy spread is insignificant during bending. We conclude that our two-stage technique not only can raise the current by at least two orders of magnitude but with appropriate tuning of the beam optics it can also minimize the associated emittance growth to less $1 \%$.

\section{SUMMARY}

In recent years, the demand for applications of electron beams with low transverse normalized emittance, short bunch length, and high peak current has increased dramatically. Most popular 
examples include high-brightness light sources [1], plasma wave accelerations [2] and linear colliders [3]. Unfortunately, space-charge at low energy prevent the generation of short bunches ( 1 <ps) directly from electron source and the commonly used process of magnetic bunch compression may unacceptably degrade the beam quality, due to significant emittance growth caused by CSR effects in the bends.

In this work we have presented a detailed beam dynamic study to improve bunch compression, consequently delivering short $(\sim 15 \mathrm{fs})$ and high peak current $(\sim 1.5 \mathrm{kA})$ bunches with minimum emittance dilution. The core idea of our scheme was to implement a two-stage solution. In the first stage, a modest compression is achieved with an innovative velocity bunch technique in which the longitudinal phase space was rotated in such a way that electrons on the tail of the bunch are faster than electrons in the bunch head. The lattice parameters were properly tuned so that to maintain a linear longitudinal phase-space after compression. In the second stage, the beam was further compressed by a conventional magnetic bunch compressor. Due to the initial VB, the chicane compressed the beam with less distortion from the CSR since the dipoles of the compressor were of lower bending angle. With the aid of numerical simulations, we showed that with this two-stage scheme the current of a $50 \mathrm{pC}$ bunch increased to a notable factor of 100 while the emittance growth reduced to $1 \%$ with appropriate tuning of the beam transport optics.

\section{ACKNOWLEDGEMENTS}

The author is very grateful to I. Ben-Zvi, M. Fedurin, Y. Jing and C. Swinson for many fruitful discussions. This work supported by the U.S. Department of Energy, contract no. DE-AC0298CH10886. 


\section{REFERENCES}

[1] P. Emma, R. Akre, J. Arthur, R. Bionta, C. Bostedt, J. Bozek, A. Brachmann, P. Bucksbaum, R. Coffee, F.-J. Decker et al., Nat. Photonics 4, 641 (2010).

[2] A. Bacci and A. R. Rossi, Nucl. Instrum. Meth. A 740, p. 42 (2014).

[3] J. Ellis and I. Wilson, Nature 409, 431 (2001).

[4] R.A. Kishek, S. Bernal, C. L. Bohn, D. Grote, I. Haber, H. Li, P. G. O'Shea, M. Reiser, and M. Walter, Physics of Plasmas 10, p. 2016 (2003).

[5] T. Ishikawa, H. Aoyagi, T. Asaka, Y. Asano, N. Azumi, T. Bizen, H. Ego, K. Fukami, T. Fukui, Y. Furukawa et al., Nat. Photonics 6, 540 (2012).

[6] Talman, Phys. Rev. Lett. 56, 1429 (1986).

[7] Ya. S. Derbenev, J. Rossbach, E. L. Saldin, and V. D. Shiltsev, Deutsches ElektronenSynchrotron, Report No. TESLA-FEL 95-05, 1995.

[8] B. E. Carlsten and T. O. Raubenheimer, Phys. Rev. E 51, 1453 (1995).

[9] M. Borland, J. W. Lewellen, S. V. Milton, "A highly flexible bunch compressor for the APS LEUTLFEL”, in proceedings of LINAC2000, Monterey,USA, August 2000.

[10] S. Di Mitri, M. Cornacchia, and S. Spampinati, Phys. Rev. Lett. 110, 014801 (2013).

[11] Y. Jing, Y. Hao, and V. N. Litvinenko, Phys. Rev. ST Accel. Beams 16, 060704 (2013).

[12] L. Serafini and M. Ferrario, AIP Conf. Proc. 581, 87 (2001).

[13] P.G. O'Shea, S.C. Bender, B.E. Carlsten, J.W. Early, D.W. Feldman, A.H. Lumpkin, R.B. Feldman, J.C. Goldstein, K.F. McKenna, R. Martineau et al., Nucl. Instrum. Meth. A 331, p. 62 (1993).

[14] X. J. Wang, X. Qiu, and I. Ben-Zvi. Phys. Rev. E 54, R3121 (1996). 
[15] M. Ferrario, D. Alesini, A. Bacci, M. Bellaveglia, R. Boni, M. Boscolo, M. Castellano, E. Chiadroni, A. Cianchi, L. Cultrera et al., Phys. Rev. Lett. 104, 054801 (2010).

[16] S. G. Anderson, P. Musumeci, J. B. Rosenzweig, W. J. Brown, R. J. England, M. Ferrario, J. S. Jacob, M. C. Thompson, G. Travish, A. M. Tremaine, and R. Yoder, Phys. Rev. ST Accel. Beams 8, 014401 (2005).

[17] H. Iijima, M. Uesaka, F. Sakamoto, T. Ueda, N. Kumagai and L. Serafini, Jpn. J. Appl. Phys. 44, 5249 (2005).

[18] P. Piot, L. Carr, W. S. Graves, and H. Loos, Phys. Rev. ST Accel. Beams 6, 033503 (2003).

[19] D. Filippetto, M. Bellaveglia, M. Castellano, E. Chiadroni, L. Cultrera, G. Di Pirro, M. Ferrario, L. Ficcadenti, A. Gallo, G. Gatti, et al., Phys. Rev. ST Accel. Beams 14, 092804 (2011).

[20] www.bnl.gov/atf

[21] K. Floetmann, ASTRA particle tracking code, http://tesla.desy.de/ meykopff

[22] M. Borland, Phys. Rev. ST Accel. Beams 4, 070701 (2001).

[23] R. Brinkmann, Y. Derbenev, and K. Flottmann, Phys. Rev. ST Accel Beams 4, 053501 (2001).

[24] P. Piot, Y. -E Sun, and K. -J. Kim, Phys. Rev. ST Accel. Beams 9, 031001 (2006).

[25] J. Zhu, P. Piot, D. Mihalcea, and C. R. Prokop, Phys. Rev. ST Accel Beams 17, 084401 (2014). 


\section{TABLES AND FIGURE CAPTIONS}

Table I: Velocity bunching beam parameters at OP for two different compression strengths. Note that the magnetic bunch compressor is turned off.

\begin{tabular}{lcc}
\hline \hline & $\mathrm{C}=1.0$ & $\mathrm{C}=4.1$ \\
\hline Bunch charge $(\mathrm{pC})$ & 50 & 50 \\
Energy $(\mathrm{MeV})$ & 116.5 & 83.2 \\
Energy spread rms & $0.02 \%$ & $0.55 \%$ \\
Emittance, $\varepsilon_{\mathrm{n}, \mathrm{x}}(\mu \mathrm{m})$ & 0.65 & 0.71 \\
Emittance, $\varepsilon_{\mathrm{n}, \mathrm{y}}(\mu \mathrm{m})$ & 0.65 & 0.71 \\
rms bunch length $(\mathrm{ps})$ & 1.13 & 0.277 \\
Laser pulse rms length $(\mathrm{ps})$ & 2.9 & 2.9 \\
Cathode rms radius $(\mathrm{mm})$ & 0.25 & 0.25 \\
Gun peak field $(\mathrm{MV} / \mathrm{m})$ & 110 & 110 \\
Gun phase $($ deg.) & -17 & -17 \\
Peak field of S1 $(\mathrm{T})$ & 0.19 & 0.19 \\
L1 accelerating field $(\mathrm{MV} / \mathrm{m})$ & 20 & 20 \\
L1 phase (deg.) & 0 & -84 \\
L2 accelerating field $(\mathrm{MV} / \mathrm{m})$ & 30 & 30 \\
L2 phase (deg.) & 0 & 0 \\
\hline
\end{tabular}

Table II: Optimum magnetic bunch compressor parameters for full compression after VB at $\mathrm{C}=4.1$ for a $50 \mathrm{pC}$ beam.

\begin{tabular}{lc}
\hline \hline Parameter & Value \\
\hline Dipole angle (deg) & 7.4 \\
Dipole length (m) & 0.19 \\
Drift between dipole 2 and 3 (m) & 0.24 \\
Drift between dipole 1 and 2 (m) & 0.32 \\
R56 at the end of chicane (cm) & -1.1 \\
\hline
\end{tabular}


FIG. 1: Overview of our accelerator beamline illustrating the solenoidal lenses ( $\mathrm{S} 1$ and $\mathrm{S} 2$ ), two linac sections (L1 and L2), quadrupole triplet and the magnetic bunch compressor (BC). Point OP depicts the end of the beamline. If otherwise stated BC is assumed to be turned off.

FIG. 2: Simulated rms bunch length (left) and corresponding compression factor (right) of a 50 $\mathrm{pC}$ beam at $\mathrm{OP}$ versus the phase of the linac L1. The right plot shows that with velocity bunching the bunch can be compressed up to a factor of 28 .

FIG. 3: Beam energy at $\mathrm{OP}$ as a function of the phase of linac $\mathrm{L} 1$ for a $50 \mathrm{pC}$ beam. Blue dots show the values for on-crest acceleration. Clearly, with velocity bunching (red dots) the final beam energy is reduced.

FIG. 4: Evolution of the transverse normalized rms emittance along the first $12 \mathrm{~m}$ of the beamline in Fig. 1 for several compression factors: $\mathrm{C}=17.4$ (red), $\mathrm{C}=9.1$ (blue) and $\mathrm{C}=3.5$ (black). The emittance without compression $(\mathrm{C}=1)$ is $0.65 \mu \mathrm{m}$.

FIG. 5: Current beam profiles at $\mathrm{OP}$ as function of the compression phase. The current distributions display asymmetric profiles with long tails which is highly correlated to the degree of compression.

FIG. 6: Longitudinal phase-space distribution after VB at two different compression factors: (a) $\mathrm{C}=27.9$ and, (b) $\mathrm{C}=3.5$. Note that at $\mathrm{C}=3.5$ phase-space nonlinearities tend to vanish suggesting that the VB method is more suitable for moderate compressions.

FIG. 7: Results from our proposed two-staged compression scheme for a $50 \mathrm{pC}$ initial round beam: (a) Emittance evolution along the beamline, (b) current distribution at OP, (c) configuration space at OP, and (d) transverse phase-space at OP. The BC angle is 7.4 deg. Note that with this scheme the peak current has increased by a notable factor of 100 and the rms bunch length reduced at fs scale (15 fs) while the associated emittance growth is near $25 \%$. 
FIG. 8: Results from our proposed two-staged compression scheme for a $50 \mathrm{pC}$ initial asymmetric (flat) beam: (a) Emittance evolution along the beamline, (b) current distribution at $\mathrm{OP}$, (c) configuration space at $\mathrm{OP}$, and (d) transverse phase-space at $\mathrm{OP}$. The $\mathrm{BC}$ angle is 7.4 deg. Note that with this scheme the peak current has increased by a factor of 100 and the rms bunch length reduced at fs scale ( $15 \mathrm{fs}$ ) while the associated emittance growth is only $1 \%$. 
Figure 1

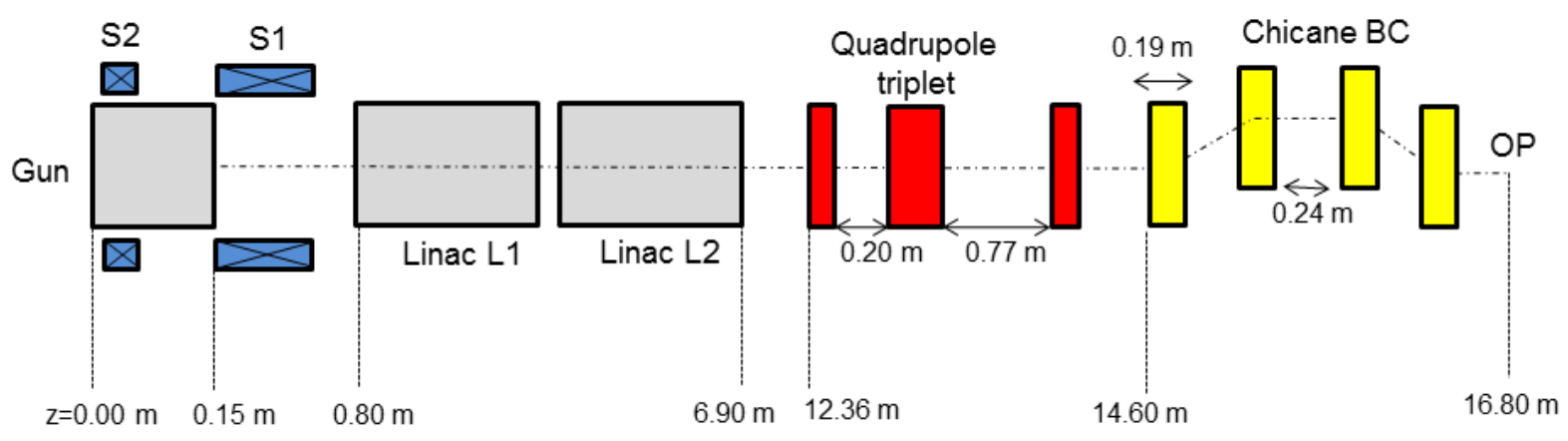


Figure 2

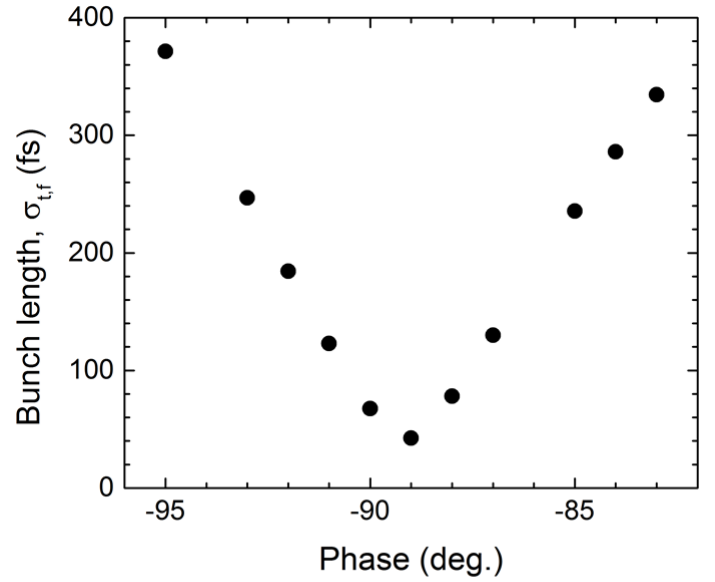

(a)

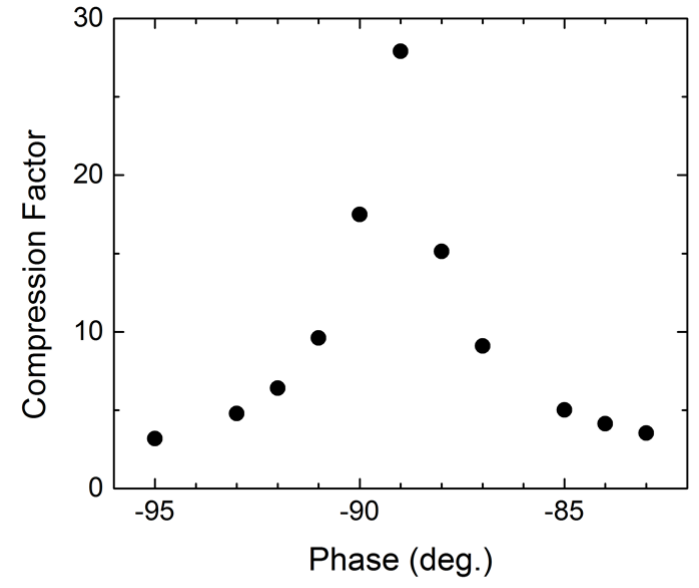

(b) 
Figure 3

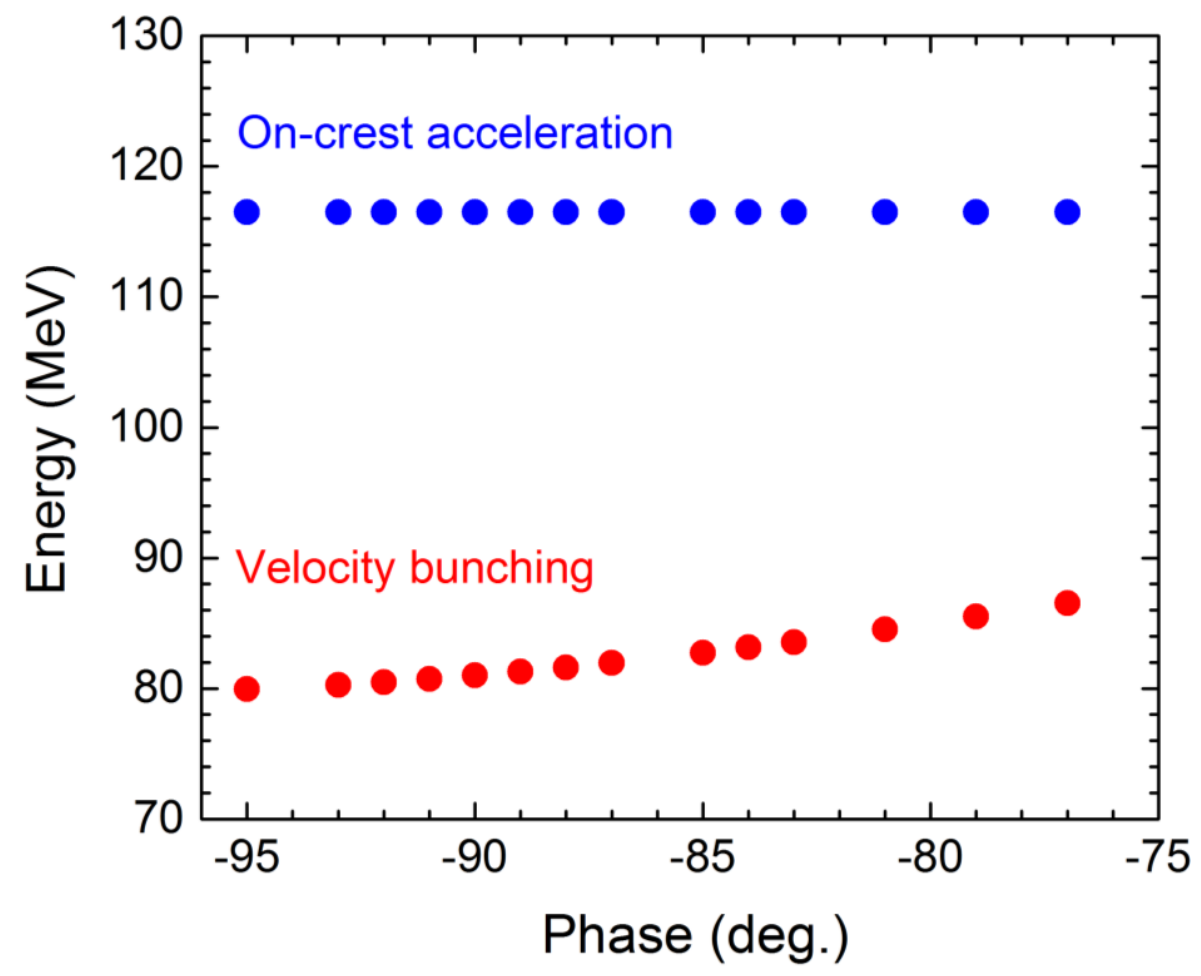


Figure 4

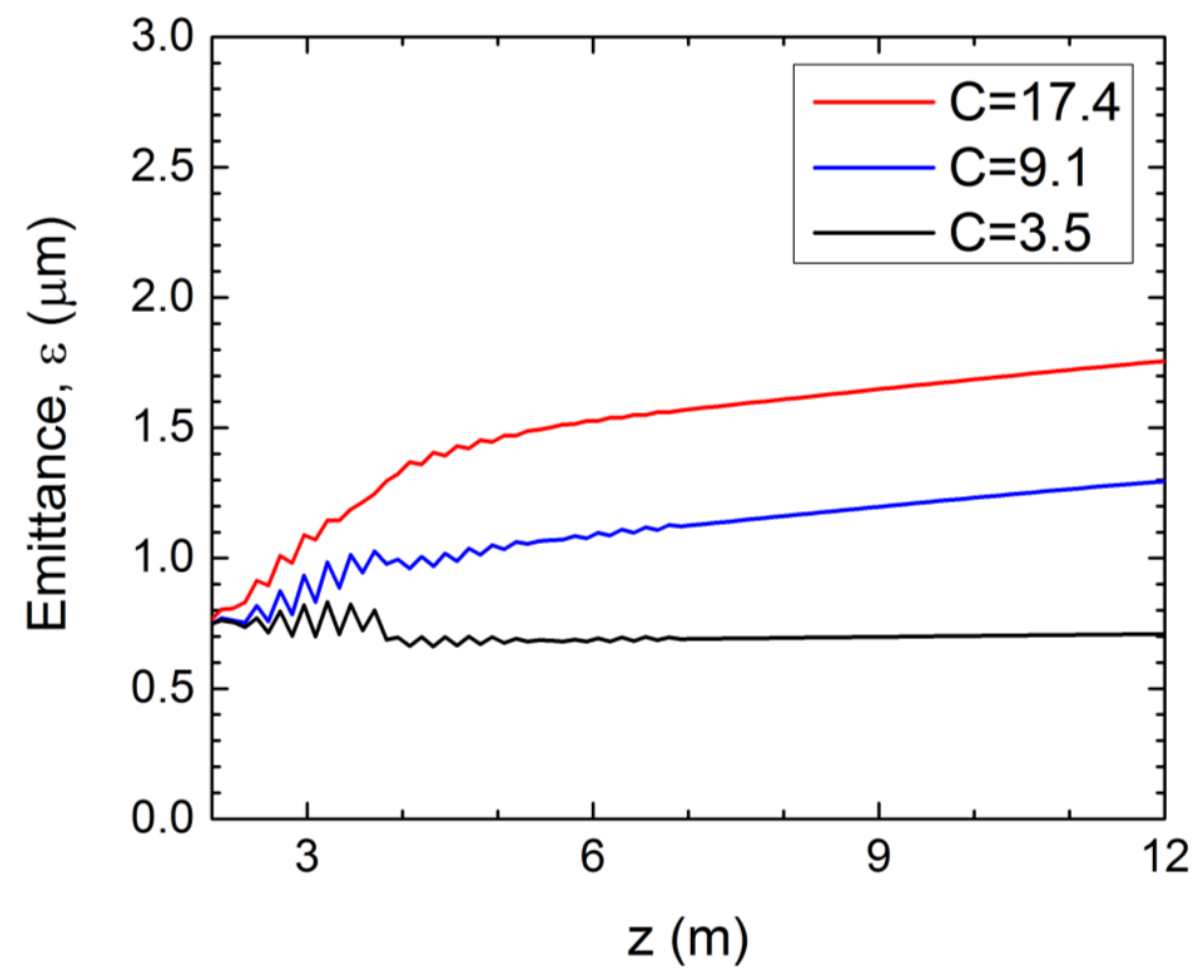


Figure 5
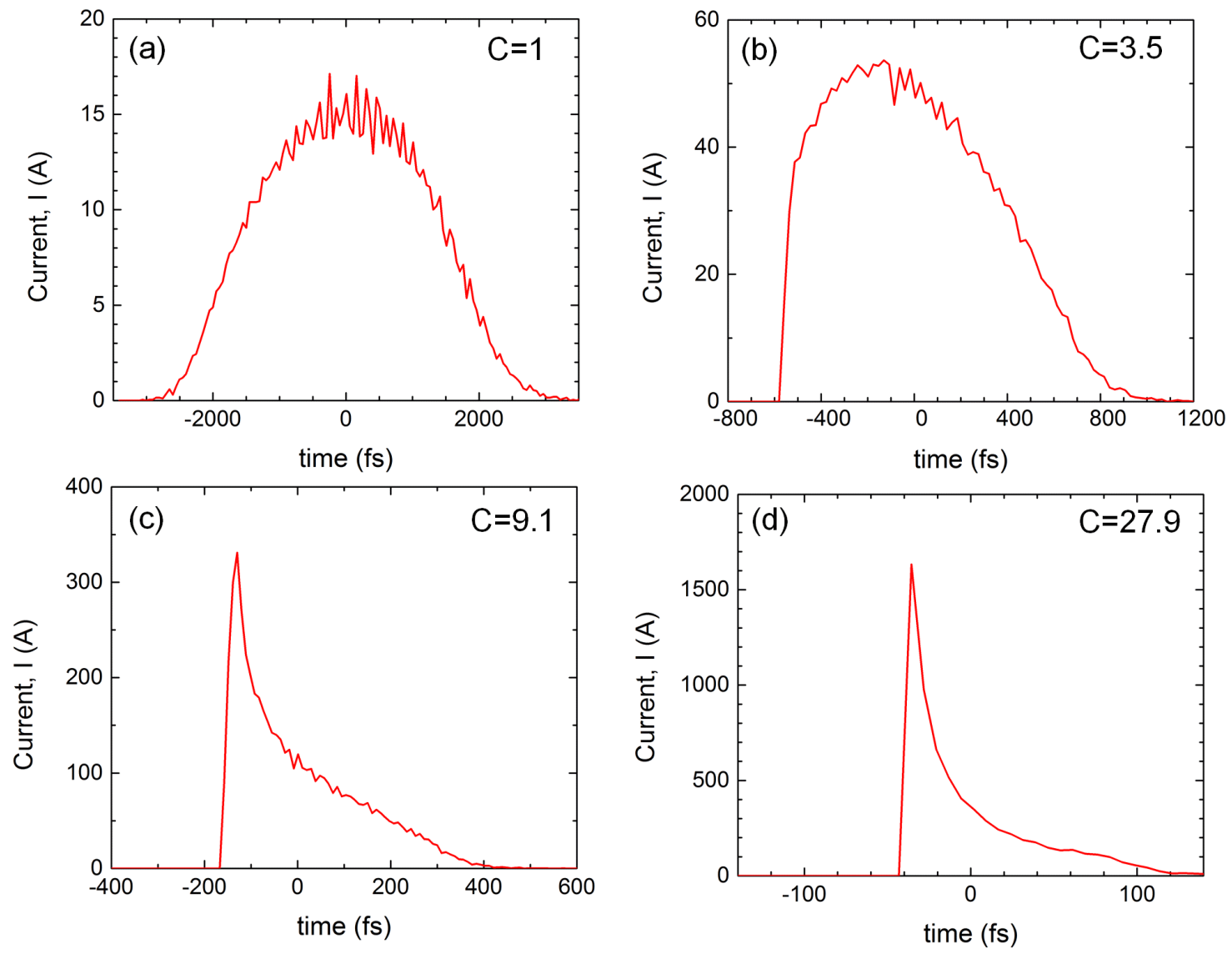
Figure 6
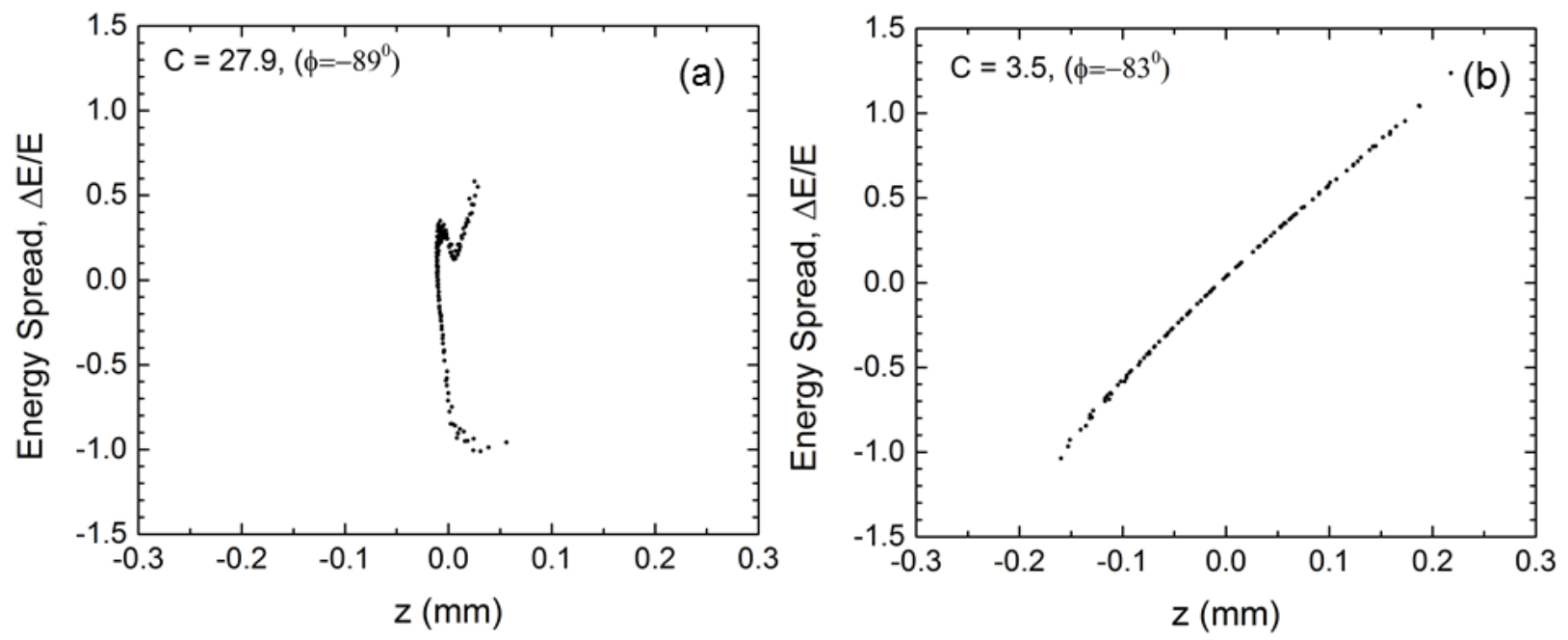
Figure 7
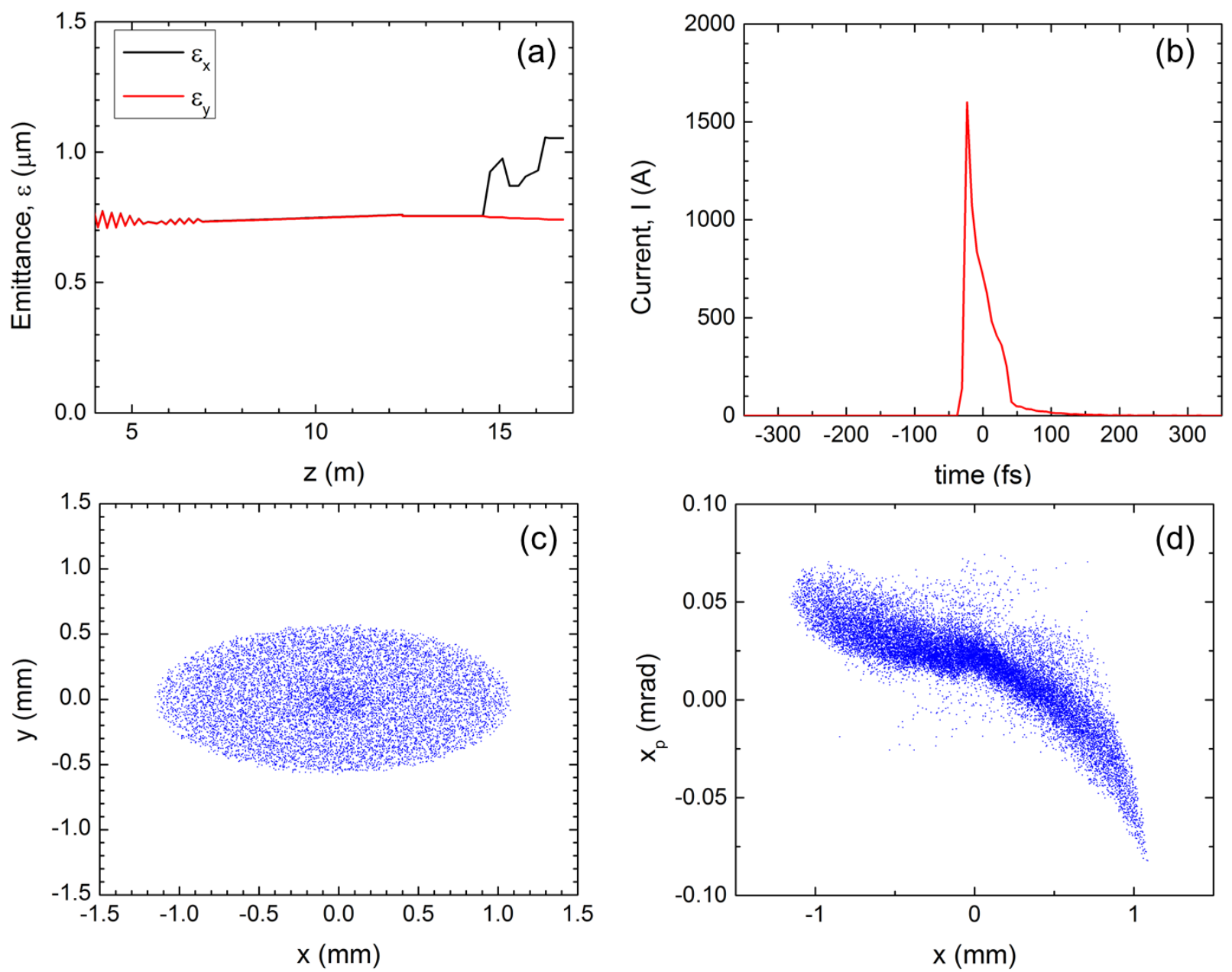
Figure 8
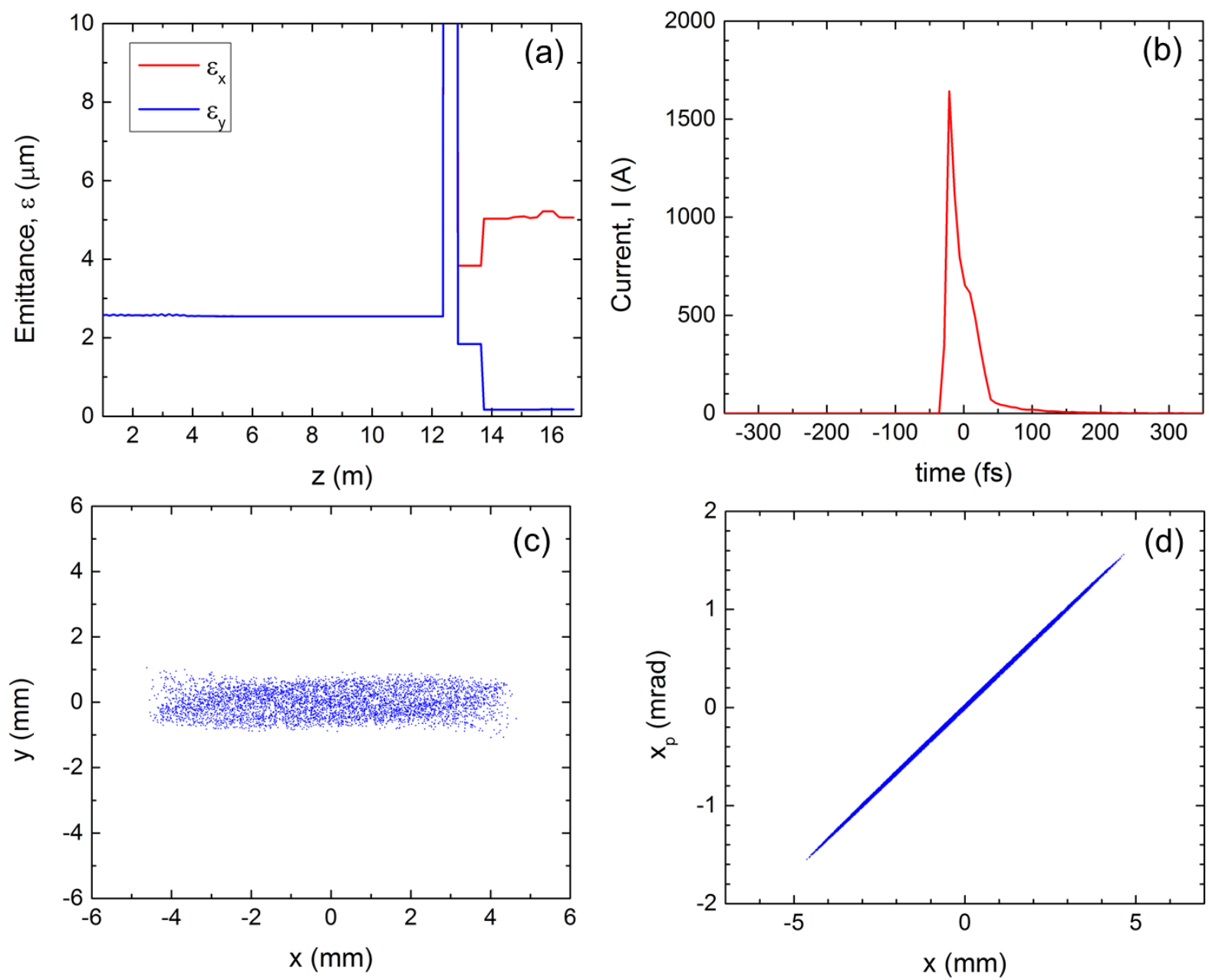